\title{
DUAL CHANNEL OCUM PROTOCOL FOR WIRELESS LANS
}

\author{
A.Chockalingam, P.Venkataram and A.Prabhakar* \\ Department of Electrical Communication Engineering \\ Indian Institute of Science, Bangalore 560012, India \\ * Indian Telephone Industries Ltd., Bangalore 560016, India
}

\begin{abstract}
Dual Channel operation of an Optimum Channel Utilisation Multiaccess (DC-OCUM) protocol using busytone concept for fully connected and homogeneous broadband wireless LANs is considered. The DC-OCUM protocol uses two independent message and busytone channel pairs. The message channels are synchronised slotted channels with slot size equal to one packet duration and the busytone channels indicate the idle or busy condition of the respective message channels. Two variations of the arrival process one without explicit rescheduling and the other with a retransmission probability for rescheduled messages are considered. The throughput performance of the DC-OCUM protocol has been estimated through a simulation model for Bernoulli arrival process and Geometric distribution of message length measured in number of packets. The performance of the protocol under two different channel selection policies - one which selects a random channel among the available idle channels and the other which chooses a random channel first and then senses - are evaluated and compared.
\end{abstract}

\section{Introduction}

Wireless networks [1] are becoming increasingly popular for local area networking applications since they eliminate the problems involved in cabling within and across buildings in wired networks like Ethernet. The future integrated services (voice, data, video) environment needs broadband networks. The latest developments in the wireless transmission technology render wireless networks the capability to carry broadband services [2]. Providing wideband network capacity using wireless medium can be accomplished by allocating a single high bandwidth channel for all the users to share through an agreed channel access algorithm. However, at high data rates multiaccess algorithms like CSMA suffer performance degradation due to increased values of the ratio between the propagation delay and the packet duration. Multichannel protocols [3] can overcome this problem by splitting a single high bandwidth channel in to a number of lower bandwidth channels each carrying a lesser data rate. Various studies by analysis and simulation have quantified the amount of performance improvement achieved in multichannel protocols applied to ALOHA, CSMA and CSMA/CD [4]-[8]. Multichannel systems offer the added advantages like increased network reliability and flexibility of system capacity expansion or reduction by simply adding or deleting individual channels. An additional feature of multichannel systems apart from channel splitting is the channel selection and rescheduling mechanism. Two types of channel selection policies for making a transmission attempt are common [3],[6]. In the first policy, sensing of all the channels is performed first and a channel is chosen randomly from the available idle channels. In the second policy, any one of the channels is selected randomly first and then the chosen channel is sensed for making a transmission attempt.

Multichannel systems in bus and ring configurations have been studied in $[9],[10]$ and a cellular mobile radio system employing multichannel CSMA is reported in [11]. Packet Radio Networks (PRN) employing several parallel multiaccess channels in multihop environment is analysed in [12]. In our earlier attempts, an Optimum Channel Utilisation Multiaccess (OCUM) protocol [13] based on an independent busytone channel concept suitable for fully connected and homogeneous PRNs has been designed and analysed. Maximum throughputs of the order of 0.8 are achieved. The effect of propagation and processing delays on the OCUM protocol performance indicating the degradation in throughput at high data rates of the order of $100 \mathrm{Mbps}$ is reported in [14]. In this paper as a prelude to study the multichannel concept applied to OCUM protocol, we present the throughput performance results of a Dual Channel system operating with OCUM protocol in a wireless LAN environment. In Section II, the basic and the Dual Channel OCUM protocol are presented. Section III describes the simulation model and section IV discusses the simulation results. Section V provides the concluding remarks indicating the scope for further investigations.

\section{OCUM Protocol}

The operation of the basic OCUM protocol [13] and the Dual Channel OCUM protocol for multiple access in a fully connected wireless LAN are described in this section.

\subsection{Basic OCUMP}

The basic OCUM protocol uses one slotted message channel ( $M$ Channel) and a separate busytone channel (B-Channel). The $M$-channel is used for transmission of preamble and data packets. The B-Channel serves two purposes. First, it serves as an ACK feedback from the destination node to the source node indicating the successful reception of the preamble following which the source node can send data packets. Second, it serves as a sensing mechanism to all the other nodes indicating the activity on the M-Channel, the knowledge of which is used to make a transmission attempt or to defer it. The messages constitute multiple data packets and each packet occupies one M-channel slot duration. Transmission attempts are made at the beginning of slot boundaries. 
When a node gets a new message arrival, it senses the BChannel. If the busytone is present on the B-Channel, it refrains from making a transmission attempt and reschedules the attempt to a later time. If the busytone is absent, it sends a preamble packet - which contains the destination node address in its address field - on the M-Channel and waits for the acknowledgment (in the form of busytone on B-Channel) from the destination node immediately in the next slot. The destina. tion node receives the preamble packet correctly and broadcasts the busytone ACK, if not more than one node had transmitted preambles during the same slot. If more than one node had sent preambles in the same slot, collision occurs and none of the destination nodes would receive correctly and thus no busytone response is broadcast. In the event of the busytone ACK being received immediately, the sender node transmits the data packets continuously on M-Channel until the entire message is sent and then wajts for a new message arrival. On the contrary, if the busytone ACK is not received, the sender node reschedules the transmission attempt to a later time. A node blocks new arrivals until the previously accepted message is successfully transmitted. The basic OCUM protocol operation is illustrated in Fig(1).
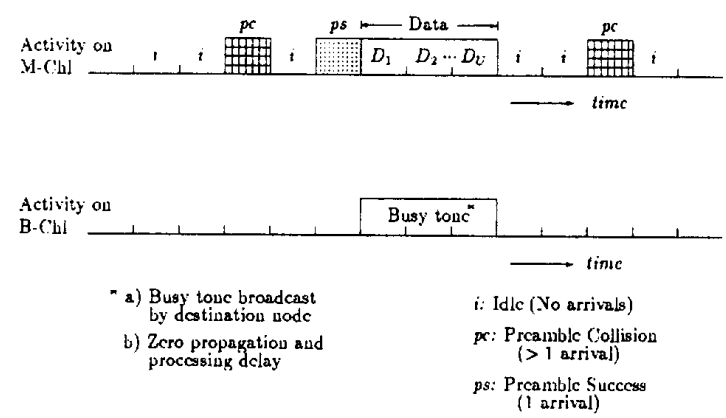

FIF 1: ONIM PROTOCOL OPERATION

\subsection{Dual Channel OCUMP}

The dual channel OCUM protocol operation is similar to the basic OCUM protocol except that each node has two separate message/busytone channel pairs and the choice of a channel pair for each attempt is made based on a channel selection policy. Two channel selection policies studied in this paper are described below.

\section{Channel Select Policy.1}

In the first policy (CSP.1), when a node gets a message arrival, it senses both B-Channels (BC-1 \& BC-2) and identifies the idle ones. If both $\mathrm{B}$-Channels are idle, then it randomly chooses one of the M-Channels (MC-1 or MC-2) with equal probability and makes the transmission attempt on the selected channel. If one of the B-Channels is busy and the other idle, it attempts on the idle M-Channel. In the event of a collision or both the BChannels being busy, the transmission attempt is rescheduled to a later time.

\section{Channel Select Policy.2}

In the second policy (CSP.2), a node on message arrival, randomly chooses any one of the B-Channels (BC-1 or BC-2) with equal probability. It then senses the chosen B-Channel and makes a transmission attempt on the corresponding $M$-Channel if the B-Channel is sensed idle and reschedules the attempt if busy.

\section{Simulation model}

In this section, we describe the simulation model devejoped to study the throughput performance of the Dual Channel OCUM protocol. The following network scenario and conditions are assumed in the model.

\subsection{Network Configuration}

1. A finite population model with ' $N$ ' number of fully connected nodes.

2. All nodes are homogeneous with two M-Channel \& BChannel transceiver pairs and a single message buffer.

3. The M-Channels are slotted to packet size and are synchronised to each other. The message length measured in number of packets is geometrically distributed with parameter ' $\mathrm{G}_{m}$ '.

4. Instantaneous busytone acknowledgment from the destination is assumed in the event of a preamble success.

5. M-Channels are assumed to be error free and packet losses if any are only due to collision.

6. Preamble success is also considered as useful in throughput computation.

7. Other wireless channel characteristics like capture effect, fading, multipath are not considered.

\subsection{Message Arrival Process}

In this simulation, two models with slightly varied assumptions on the arrival process are studied.

1. In model.1 arrival process, the arrivals at the beginning of each slot are assumed to be statistically independent of the previous history of arrivals and channel events. In otherwords, the rescheduled messages are assumed to be regenerated afresh along with new arrivals with Bernoulli rate ' $\lambda$ ' arrivals per slot at each node.

2. In model. 2 arrival process, new arrivals enter each node at Bernoulli rate ' $\lambda$ ' and the rescheduled messages are reattempted after a random number of slots which is geometrically distributed with parameter ' $\mathrm{G}_{r}$ '. In otherwords, the nodes with resheduled messages will make an attempt in a slot with probability ' $G_{r}$ ' and defer the attempt with probability $\left(1-G_{r}\right)$.

A simulation program has been written to implement the above models of the Dual Channel OCUM protocol. The different input parameters like $N, \lambda, G_{m}, G_{r}$, type of arrival process (model.1 or model.2), and channel select policy (CSP.1 or CSP.2) which characterise the simulation model are read from the INPUT.PARAM file. The simulation program is written in a modular fashion using various functional subprograms like Bernoulli arrival generator (ber_msg_arr), geometric message length generator (gen_msg_Jen), rescheduling delay generator (gem_retx_dly), 
channel select routines (chl_sel_plcy_l and chl_sel_plcy_2). The protocol performance measures like the system throughput, idle rate, collision rate and reject rate are estimated.

\section{Results and Discussion}

The throughput performance of the Dual Channel OCUM protocol under model. 1 arrival process for $\mathrm{G}_{m}=0.2$ and $\mathrm{N}=10$, 20 and 50 along with the single channel OCUM protocol performance is shown in Fig(2). At low arrival rates the throughput is less due to high channel idle rates. Maximum throughputs of the order of 0.8 per channel are achieved at $\frac{1}{N}$ arrival rates and for arrival rates beyond, the protocol shows negative slope throughput characteristics like other random access protocols like ALOHA, S-ALOHA and CSMA. This degradation in the throughput at high arrival rates is due to increased collision rates as depicted in Fig(3). The problem of throughput degradation at high arrival rates is overcome in the scheme with model. 2 arrival process where a retransmission probability ' $\mathrm{G}_{r}$ ' is assigned for the rescheduled traffic. This result is illustrated in Fig(4) which shows the throughput performance of the DC-OCUM protocol with rescheduling probability $G_{r}=0.05$ at $N=20$ and $G_{m}=0.2$ for both channel select policies CSP.1 and CSP.2. At high arrival rates both channel select policies offer saturated throughputs of the order of 0.8 per channel. However, channel select policy CSP.1 is found to perform marginally better than CSP.2. This superiority of CSP.1 over CSP.2 is well explained in Fig(5). The per channel idle rate is less for CSP.1 than compared to CSP.2 whereas the per channel collision rate is more for CSP.1 than CSP.2. The net effect on the throughput is in favour of CSP.1 since the difference between the respective idle rates is higher than the difference in the collision rates. Fig(5) also shows the reject rate of newly arriving messages at each node, which at high arrival rates is found to increase as close as to 1 . In Fig(6) the throughput performance of the DC-OCUM protocol for $\mathrm{N}=$ $20, \mathrm{Gr}=0.05$ and $\mathrm{N}=5, \mathrm{Gr}=0.2$ at $\mathrm{Gm}=0.2$ are plotted.

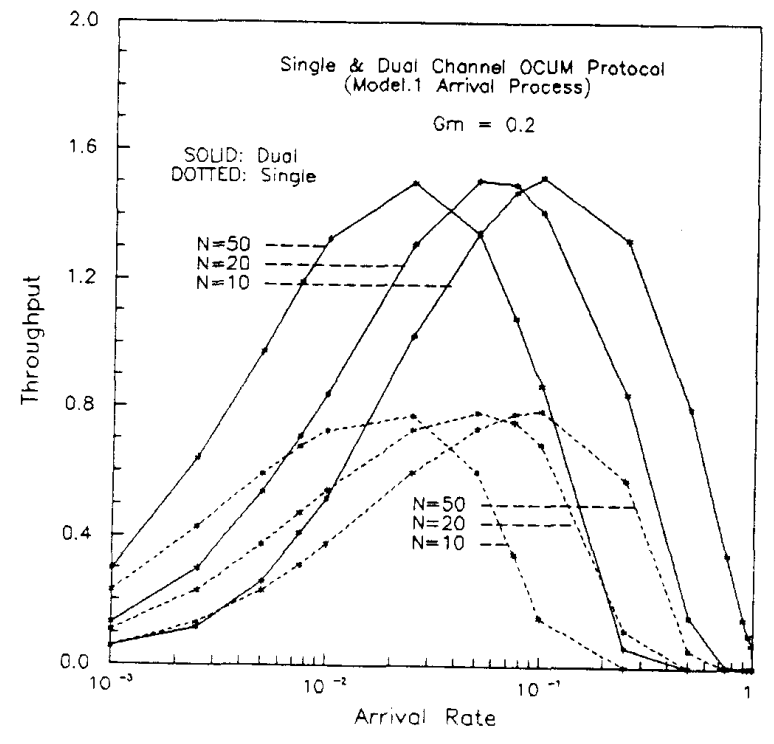

FIG.2: Single \& Dual Chonnel OCUM Protocol performance under Model.1 Arrival Process ot diff. "N'values

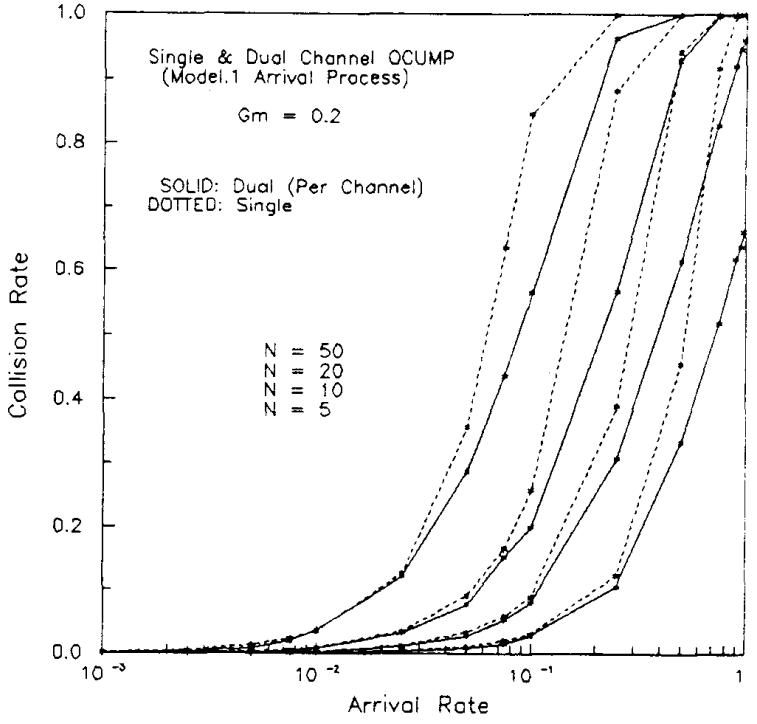

FIG.3: Collision (per channel) performance of Single \& Duol Channel OCUM Protocol under Model. 1 Arrival Process

\section{Conclusion}

We presented the throughput performance of a Dual Channel Optimum Channel Utilisation Multiaccess protocol employing two separate message/busytone channel pairs. The throughput estimation was done using simulation models for two variations of the arrival process one without explicit rescheduling and the other with rescheduling using a finite retransmission probability. The unstable behavoiur of the protocol observed at high arrival rates under the model without rescheduling is overcome in the model with rescheduling. Two channel select policies were investigated and the policy with idle channel choice is found to

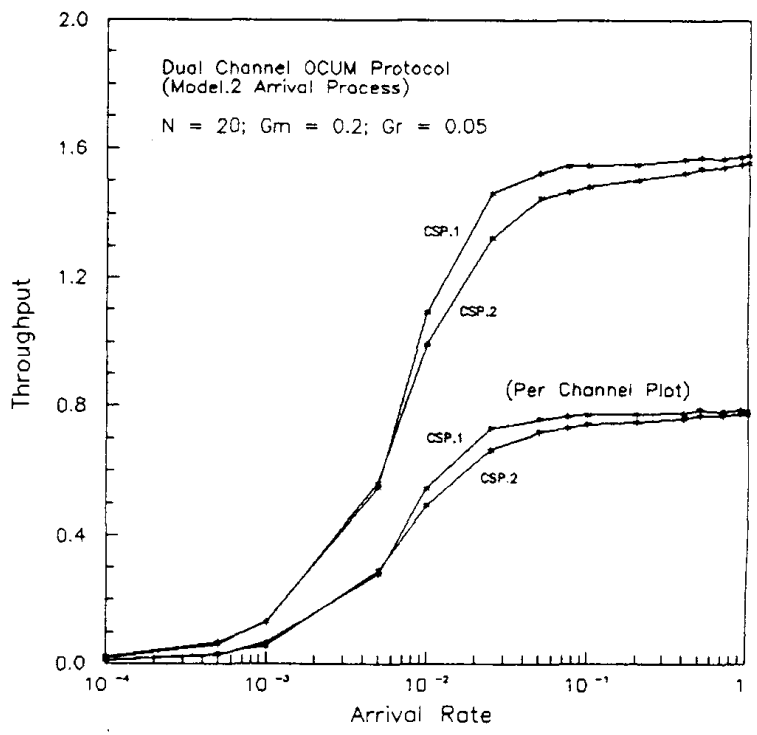

FIG.4: Duol Channel OCUM Protocol throughput performance under Model.2 Arrival Process 
perform better than the random channel choice. We further propose to study the delay characteristics of the Dual Channel OCUM protocol. Also, the study of a more generalised $n$-channel $(n>2)$ system with finite message buffer capacity $(>1)$ at all nodes is envisaged.

\section{Acknowledgment}

The first author would like to thank Shri.B.S.Subbaramaiah, Indian Telephone Industries Ltd., Bangalore, India for his encouragement and help to carry out this work.

\section{References}

[1] Dale Buchholz, Paul Odlyzko, Mark Taylor and Richard White, "Wireless In-Building Network Architecture and Protocols," IEEE Network Magazine, Vol.5, No.6, pp.3138, November 1991.

[2] M.Carloni, T.S.Chu, M.J.Gans and P.W.Wolniansky, "A 2.5 Gigabit 23 mile Radio Link for LuckyNet," Proc. IEEE GLOBECOM'91, Vol.2, Phoenix, AZ, December 1991.

[3] M.Ajmone Marsan and D.Roffinella, "Multichannel local Area Network Protocols," IEEE Jl. Sel. Areas Commun., Vol.SAC-1, No.5, pp.885-897, November 1983.

[4] G.L.Choudhury and S.S.Rappaport, "Diversity ALOHA A Random Access Scheme for Satellite Communications," IEEE Trans. Commun., Vol.COM-31, No.3, pp.450-457, March 1983.

[5] M.Ajmone Marsan,

P.Camarda and D.Roffinella, "Throughput and Delay Characteristics of Multichannel CSMA/CD Protocols," Proc. IEEE GLOBE COM'83, pp.1147-1151, San Diego, CA, November 1983.

[6] H.Okada, Y.Nomura and Y.Nakanishi, "Multichannel CSMA/CD method in Broadband Bus Local Area Networks," Proc. IEEE GLOBECOM'84, Vol.2, pp.642-647, Atlanta, GA, November 1984 .

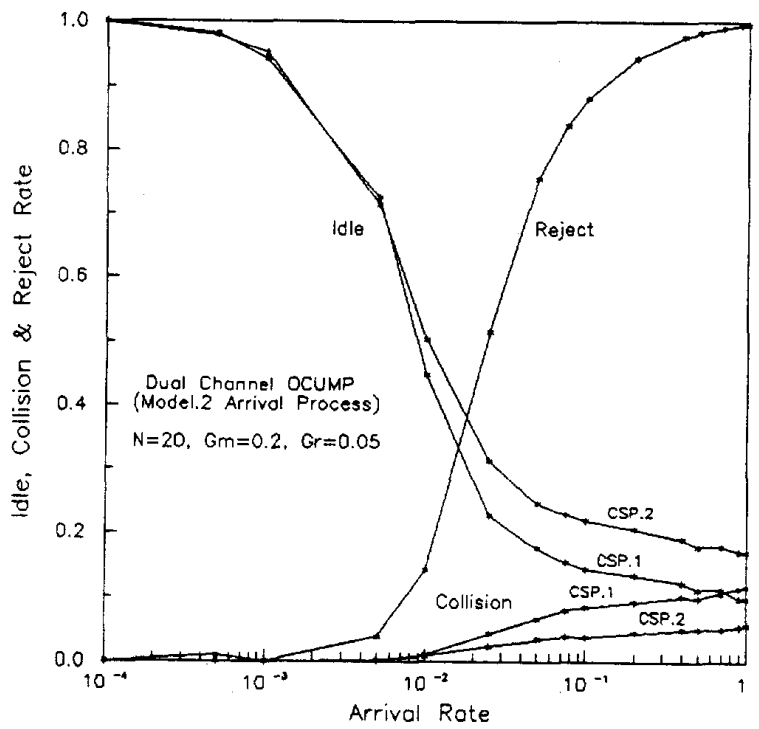

FIG.5: Idle, Collision (per channel) \& reject rate performance of DC-OCUM Protocol under Model. 2 Arrival Process

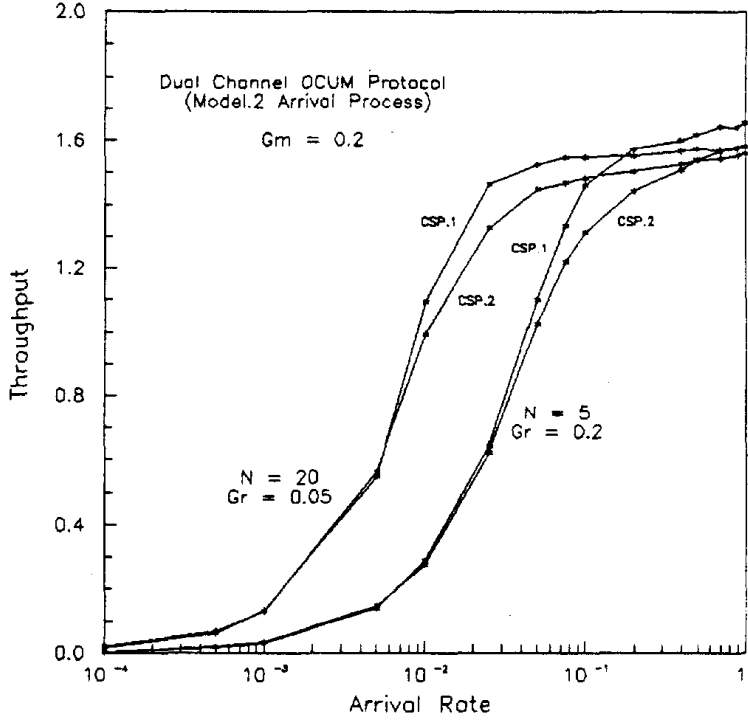

FIG.6: DC-OCUM Protocol throughput periormance under Model.2 Arrival Process at diff. values of $N \& G r$

[7] M.Ajmone Marsan and P.Camarda, "The Effect of Acknowledgment Traffic on the performances of Multichannel CSMA/CD protocols," Proc. IEEE INFOCOM'85, pp.410418, Washington D.C., March 1985.

[8] T.Todd, "Throughput in Slotted Multichannel CSMA/CD systems," Proc. IEEE GLOBECOM'85, New Orleans, December 1985.

[9] A.Ganz and I.Chlamtac, "Analysis of Multiple-Bus synchronous and asynchronous communication systems," Perform. Eval., Vol.5, No.1, pp.1-13, 1985

[10] Joseph C.Palais and T.I.Yuk, "Multichannel buffer insertion ring LAN," Computer Communications, Vol.14, No.8, pp.443-450, October 1991.

[11] Newman D.Wilson and Stephen S.Rappaport, "Cellular Mobile Radio using Multiple Channel CSMA," Proc. IEEE INFOCOM'85, pp.370-376, Washington D.C., March 1985.

[12] Nachum Sacham and Peter J.B.King, "Architectures and performance of Multichannel Multihop Packet Radio Networks," IEEE Jl. Sel. Areas in Commun., Vol.SAC-5, No.6, pp.1013-1025, July 1987.

[13] A.Chockalingam, P.Venkataram and A.Prabhakar, "Design of an Optimal Channel Utilisation Multiaccess Protocol for Packet Radio Networks," submitted to journal IETE.

[14] A.Chockalingam, P.Venkataram and A.Prabhakar, "Effect of propagation and processing delays on the throughput performance of a class of multiaccess protocol for High Speed Radio LANs," Proc. IEEE GLOBECOM'91, Vol.2, pp.906910, Phoenix, AZ, December 1991. 